\title{
Therapeutic effects of the combined androgen blockade therapy versus luteinizing hormone-releasing hormone analog monotherapy in patients with hormone naïve metastatic prostate cancer: a multi-institutional comparative analysis
}

\author{
Takuma Narita ${ }^{1}$, Shingo Hatakeyama ${ }^{1}$, Shintaro Narita ${ }^{2}$, Masahiro Takahashi ${ }^{3}$, Toshihiko Sakurai ${ }^{4}$, \\ Sadafumi Kawamura ${ }^{5}$, Senji Hoshi ${ }^{6}$, Jiro Shimoda ${ }^{7}$, Toshiaki Kawaguchi ${ }^{8}$, Shigeto Ishidoya ${ }^{9}$, \\ Koji Mitsuzuka ${ }^{3}$, Yoichi Arai ${ }^{5}$, Akihiro Ito ${ }^{3}$, Norihiko Tsuchiya ${ }^{4}$, Tomonori Habuchi ${ }^{2}$, Chikara Ohyama ${ }^{1}$ \\ ${ }^{1}$ Department of Urology, Hirosaki University School of Medicine, Hirosaki, Japan; ${ }^{2}$ Department of Urology, Akita University School of Medicine, \\ Akita, Japan; ${ }^{3}$ Department of Urology, Tohoku University School of Medicine, Sendai, Japan; ${ }^{4}$ Department of Urology, Yamagata University School \\ of Medicine, Yamagata, Japan; ${ }^{5}$ Department of Urology, Miyagi Cancer Center, Natori, Japan; ${ }^{6}$ Department of Urology, Yamagata Prefectural \\ Central Hospital, Yamagata, Japan; ${ }^{7}$ Department of Urology, Iwate Prefectural Isawa Hospital, Oshu, Japan; ${ }^{8}$ Department of Urology, Aomori \\ Prefectural Central Hospital, Aomori, Japan; ${ }^{9}$ Department of Urology, Sendai City Hospital, Sendai, Japan \\ Contributions: (I) Conception and design: T Narita, S Hatakeyama; (II) Administrative support: S Hatakeyama, S Narita, C Ohyama; (III) Provision of \\ study materials or patients: All authors; (IV) Collection and assembly of data: All authors; (V) Data analysis and interpretation: T Narita, S Hatakeyama; \\ (VI) Manuscript writing: All authors; (VII) Final approval of manuscript: All authors. \\ Correspondence to: Shingo Hatakeyama, MD. Department of Urology, Hirosaki University Graduate School of Medicine, 5 Zaifu-chou, Hirosaki 036- \\ 8562, Japan. Email: shingoh@hirosaki-u.ac.jp.
}

Background: The clinical benefit of the combined androgen blockade (CAB) therapy over luteinizing hormone-releasing hormone analog (LH-RHa) monotherapy for hormone naïve metastatic prostate cancer (mHNPC) is unclear. Therefore, we retrospectively compare the effectiveness of CAB with the LH-RHa monotherapy on the prognosis of Japanese patients with mHNPC.

Methods: We retrospectively evaluated the prognosis of 517 patients diagnosed with mHNPC between August 2001 and May 2017. The patients' data were obtained from the Michinoku Urological Cancer Research Group database and Hirosaki University-related hospitals. Patients were divided into the CAB and LH-RHa monotherapy groups based on primary androgen deprivation therapy (ADT). Overall survival (OS), cancer-specific survival (CSS), and castrate-resistant prostate cancer-free survival (CRPC-FS) were compared between the two groups using the Kaplan-Meier curve analysis. Inverse probability of treatment weighting (IPTW)-adjusted Cox hazard proportional analyses was performed to investigate the effect of primary ADT on oncological outcomes.

Results: The median age was 73 years old. The numbers of patients in the CAB and LH-RHa monotherapy groups were 447 and 70, respectively. The Kaplan-Meier curve analysis showed no significant differences in either 5 -year OS (56.7\% vs. 52.5\%, P=0.277), CSS (61.1\% vs. 56.4\%, $\mathrm{P}=0.400)$, and CRPCFS (33.1\% vs. 31.1\%, $\mathrm{P}=0.529)$ between the groups. IPTW-adjusted multivariate Cox hazard proportional analyses showed no significant differences in OS, CSS, and CRPC-FS between the two groups.

Conclusions: No significant differences in oncological outcomes were observed between the CAB and LH-RHa monotherapy groups in patients with mHNPC.

Keywords: Metastatic prostate cancer; luteinizing hormone-releasing hormone analog (LH-RHa); combined androgen blockade (CAB); bicalutamide

Submitted Jun 01, 2020. Accepted for publication Nov 17, 2020.

doi: 10.21037/tau-20-966

View this article at: http://dx.doi.org/10.21037/tau-20-966 


\section{Introduction}

Prostate cancer is the common malignancy worldwide. In Japan, about $10 \%$ of patients with prostate cancer have distant metastases (1-3). For half a century, the standard for the initial treatment of hormone naïve metastatic prostate cancer (mHNPC) has been androgen deprivation therapy (ADT) (4). ADT includes orchiectomy, luteinizing hormone-releasing hormone analog (LH-RHa) therapy, and combined androgen blockade (CAB) including LH-RHa together with anti-androgen agents such as bicalutamide. Several guidelines do not recommend CAB therapy for patients with $\mathrm{mHNPC}$ as a first-line (5-7) therapy because no level 1 evidence supports the superiority of $\mathrm{CAB}$ as a primary treatment for mHNPC. In contrast, nearly $70 \%$ of mHNPC patients in Japan were treated initially with $\mathrm{CAB}(8)$ because a large-scale retrospective study suggested the advantage of using $\mathrm{CAB}$ to treat Japanese mHNPC. Nevertheless, there is no conclusive evidence suggesting which treatment is the optimal one as a baseline ADT for mHNPC (5-7). Although Japanese Urology Association (JUA) recommends $\mathrm{CAB}$ as one of the standard treatments (9), clinical benefits of CAB over the LH-RHa monotherapy are not understood.

In this study, we retrospectively compared the therapeutic effect of $\mathrm{CAB}$ with that of LH-RHa monotherapy as a primary treatment for $\mathrm{mHNPC}$. We present the following article in accordance with the STROBE reporting checklist (available at: http://dx.doi.org/10.21037/tau-20-966).

\section{Methods}

\section{Study design and ethical statement}

We performed the present retrospective, multicenter study in accordance with the ethical standards outlined in the Declaration of Helsinki (revised in 2013). The ethics review boards of the Hirosaki University School of Medicine (authorization number: 2017-089) and all hospitals approved the protocol.

\section{Setting and participants}

We evaluated data of patients with mHNPC who were initially treated with ADT obtained from multiple databases that included Michinoku Japan Urological Cancer Study Group database (from Hirosaki University Hospital, Akita University Hospital, Tohoku University Hospital, Yamagata University Hospital, Miyagi Cancer Center,
Yamagata Prefectural Central Hospital, Iwate Prefectural Isawa Hospital, Aomori Prefectural Central Hospital, and Sendai City Hospital) and from Hirosaki University-related hospitals' databases (from National Hospital Organization Hirosaki Hospital, Odate Municipal General Hospital, Mutsu General Hospital, Tsugaru General Hospital, and Aomori Rosai Hospital) between January 2001 and March 2019 (10-15). We defined mHNPC patients as prostate cancer patients with bone and/or distant lymph node metastasis and/or visceral metastases. Patients who received as initial treatment only the LH-RHa (leuprorelin, goserelin acetate, or degarelix) were placed into the $\mathrm{LH}-$ $\mathrm{RHa}$ monotherapy group. CAB group was defined as a group of patients who received an initial treatment of LH$\mathrm{RHa}$ combined with an anti-androgen medication such as bicalutamide.

\section{Evaluation of variables}

We analyzed the following parameters: patients' age, Eastern Cooperative Oncology Group performance status (ECOG-PS), initial prostate-specific antigen (iPSA) levels at the time of cancer diagnosis, Gleason score (GS), extent of disease (EOD), and CHAARTED highvolume disease at the time of diagnosis. EOD were used to evaluate the extent of bone metastasis, the grades were EOD 0, normal or abnormal due to benign bone disease; EOD 1, <6 metastases; EOD 2, 6-20 metastases; EOD 3, $>20$ metastases but not a superscan; and EOD 4, superscan. CHAARTED high-volume disease was defined as the presence of visceral metastases or bone metastasis $\geq 4$ with at least one bone metastasis must be present outside the vertebral bodies and pelvis in accordance with the CHAARTED study (16). Metastatic status was detected with computed tomography and bone scintigraphy before ADT. We also investigated the progression to castrateresistant prostate cancer (CRPC), treatment history with docetaxel (DTX), abiraterone acetate (AA) and/or enzalutamide (ENZ), estramustine, and bone-modifying agents (BMA) during ADT treatment. The indication of these treatments according to the personal choice of the physician in charge of the case. The definition of CRPC in this study was based on the recommendations made by the prostate cancer clinical trial working group (17).

\section{Treatment protocol}

All of the participating patients were treated initially with 
ADT and/or BMA. After CRPC diagnosis, alternative antiandrogen therapy, estramustine, chemotherapy (DTX or $\mathrm{CBZ}$ ), AA or ENZ were applied as treatment according to the personal choice of the physician in charge of the case.

\section{Statistical analysis}

Statistical analysis of data was performed using SPSS software package version 24.0 (SPSS, Inc., Chicago, IL, USA), and R 3.4.3 (The R Foundation for Statistical Computing, Vienna, Austria). Categorical variables were compared using either Fisher's exact test or the $\chi^{2}$ test. Quantitative variables were expressed as median with interquartile range and compared statistically using the Mann-Whitney $\mathrm{U}$ test. $\mathrm{P}$ values $<0.05$ were considered as statistically significant. Patients with missing data were excluded from the analysis.

We analyzed overall survival (OS), which was defined as the time from start of $\mathrm{ADT}$ until all-cause death or last patient contact. Cancer-specific survival (CSS) was defined as the time from start of ADT until cancer death or last patient contact. CRPC-FS was defined as the time from start of ADT until progression toward CRPC. In addition, the PSA best response rate was assessed. Patients with missing PSA nadir data were excluded from the PSA best response rate analysis. PSA best response rate was defined as (iPSA - nadir PSA)/(iPSA). Oncological outcomes including OS, CSS, and CRPC-FS were investigated using the Kaplan-Meier method and compared with the logrank test. We performed a Cox hazard proportional analysis using inverse probability of treatment weighting (IPTW) method to investigate the oncological outcomes for $\mathrm{CAB}$ and LH-RHa monotherapy groups considering background differences between the two groups (18). Variables included in the IPTW analysis were as follows: age, iPSA, ECOGPS, GS, EOD, CHAARTED classification, treatment with DTX, AA and/or ENZ, estramustine, BMA.

\section{Results}

\section{Baseline characteristics}

We evaluated data of 809 Japanese patients with mHNPC who were initially treated with ADT obtained from multiple databases that included Michinoku Japan Urological Cancer Study Group database $(\mathrm{n}=667)$ and from Hirosaki University-related hospitals' databases $(\mathrm{n}=142)$. We excluded 292 out of 809 participants because of either insufficient data $(n=213)$, or surgical castration monotherapy and antiandrogen monotherapy performed $(n=79)$. After all the exclusion criteria were applied, we used data from 517 cases (CAB group had 447 cases, LH-RHa monotherapy group had 70 cases) in this study. Also, we excluded 60 cases for the CRPC-free survival (CRPC-FS) analysis $(\mathrm{n}=457)$ because of the lack of data (Figure 1).

Baseline patients' characteristics are shown in Table 1. The median age of patients was 73 years. The patient proportions of $\mathrm{GS} \geq 8, \mathrm{EOD} \geq 2$, CHAARTED high volume disease were $89 \%, 56 \%$, and $63 \%$, respectively. 303 patients developed CRPC and 160 patients died within a median follow-up period of 28 months. There were no significant differences between the two groups either in age, iPSA, GS, EOD, CHAARTED high-volume disease, or progression to CRPC. In contrast, there were significant differences in ECOG-PS $(\mathrm{P}=0.047)$, treatment with DTX $(\mathrm{P}=0.004)$, treatment with BMA $(\mathrm{P}=0.020)$, and follow-up period $(\mathrm{P}<0.001)$.

\section{Oncological outcomes}

The Kaplan-Meier curve analysis showed no significant differences in either 5 -year OS (Figure $2 A, 56.7 \%$ vs. $52.5 \%$, $\mathrm{P}=0.277$ ), CSS (Figure $2 B, 61.1 \%$ vs. $56.4 \%, \mathrm{P}=0.400$ ), or CRPC-free survival (Figure 2C, 33.1\% vs. 31.1\%, $\mathrm{P}=0.529$ ) between the CAB and LH-RHa monotherapy groups. There is no significant difference in PSA best response rate (median: $99.5 \%$ vs. $99.8 \%, \mathrm{P}=0.15$, respectively, Figure $\mathrm{S} 1$ ).

\section{Uni- and multivariate analyses for prognosis}

Univariate Cox hazard proportional analysis showed that CAB was not a significant predictor for OS, CSS, and CRPC-FS (Table 2). IPTW-adjusted multivariate Cox hazard proportional analyses showed no significant difference between $\mathrm{CAB}$ and LH-RHa monotherapy groups in either OS $(\mathrm{P}=0.132$, HR 0.63, 95\% CI, 0.38-1.06), CSS $(\mathrm{P}=0.384$, HR 0.75 , 95\% CI, 0.41-1.36), or CRPC-FS $(\mathrm{P}=0.138$, HR $0.71,95 \%$ CI, 0.47-1.07) (Figure 3).

\section{Discussion}

In this study, we compared the clinical outcomes of between two treatments: CAB and LH-RH monotherapy in the real-world practice in Japan and observed no statistically significant differences in either OS, CSS, or CRPC-FS 


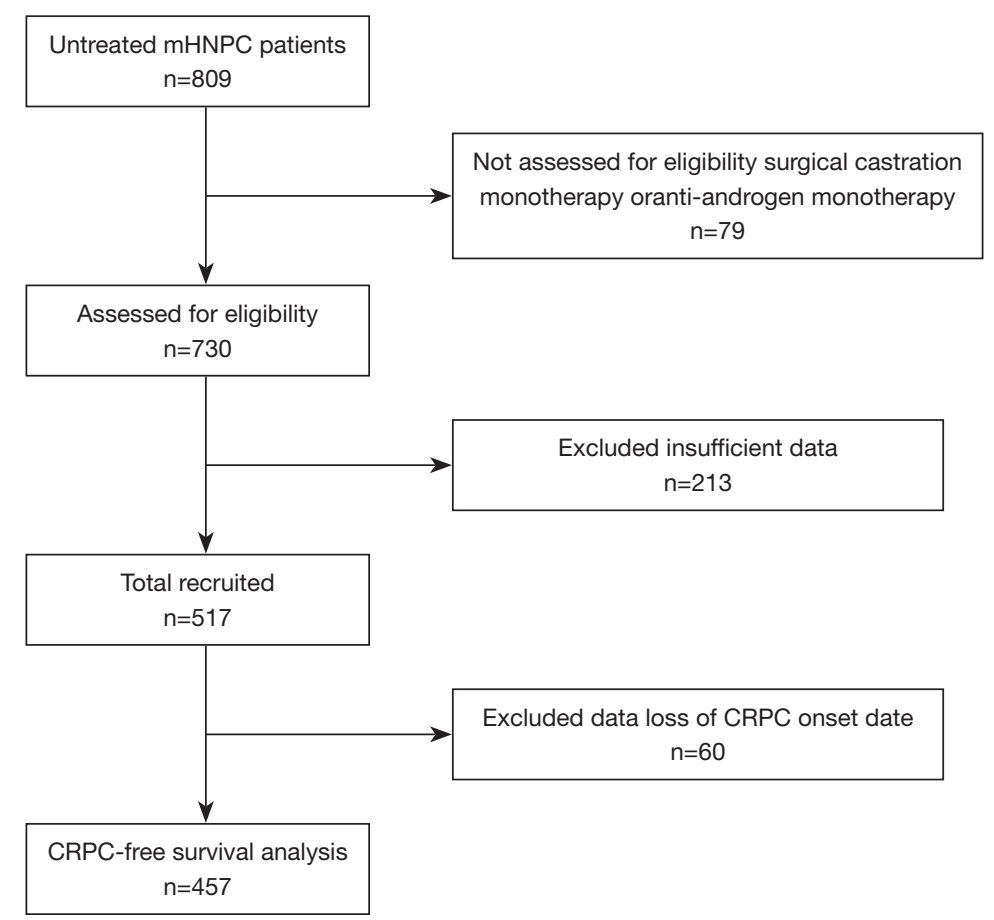

Figure 1 Flow diagram for recruitment. The flow of study identification and exclusion. mHNPC, monotherapy for hormone naïve metastatic prostate cancer; CRPC, castrate-resistant prostate cancer.

between the two groups. Although several studies suggested the potential association between the racial differences and the efficacy of ADT $(19,20)$, our results showed similar outcomes with those demonstrated for the patients from Western countries $(21,22)$.

Previous meta-analysis comparing $\mathrm{CAB}$ and LH-RHa monotherapy for patients with mHNPC $(6,821$ men in 21 randomized trials) showed no significant differences in OS (21). Another meta-analysis (8,275 men in 27 randomized trials) showed that $\mathrm{CAB}$ may improve five-year OS up to $2.9 \%$ as compared with LH-RHa monotherapy in advanced prostate cancer $(\mathrm{P}=0.005)$. However, while the participants of this meta-analysis included $12 \%$ of patients with locally advanced prostate cancer, there was no subgroup of patients with mHNPC included in this analysis (22). Due to the lack of evidence of CAB effectiveness for mHNPC treatment, several guidelines in Western countries do not recommend CAB for mHNPC as a first-line therapy (5-7). Our observations supports the guidelines recommendations used Western countries.

The randomized controlled trials (RCT) conducted in Japan failed to demonstrate the superiority of $\mathrm{CAB}$ in patients with mHNPC (23). Also, in the largest retrospective study (J-CaP study) conducted in Japan in 2018, the therapeutic effect of LH-RHa monotherapy and $\mathrm{CAB}$ was examined by propensity score matching, but the superiority of $\mathrm{CAB}$ in $\mathrm{mHNPC}$ was not proven in subgroup analysis (24). Therefore, there is no high-level evidence to support the superiority of CAB over the LHRHa monotherapy for men with mHNPC. Our results are in agreement with these studies conducted in Japan.

Why is $70 \%$ of mHNPC patients treated with $\mathrm{CAB}$ in Japan (8) without strong evidence of the superiority of CAB? It has to be debated why clinicians in Japan support the clinical benefits of CAB. First, clinicians may prescribe an anti-androgen agent combined with LH-RHa as an initial treatment to prevent testosterone "flare-up" phenomenon. It is believed that LH-RH agonists cause a transient rise in serum testosterone levels, and thereby induce "clinical flare" such as prostate cancer growth, increase bone pain, voiding problems, and spinal cord compression $(25,26)$. European Association of Urology guideline states, "Offer initial shortterm administration of antiandrogens to mHNPC patients treated with a LH-RH agonist to reduce the risk of the 
Table 1 Clinical characteristics of patients

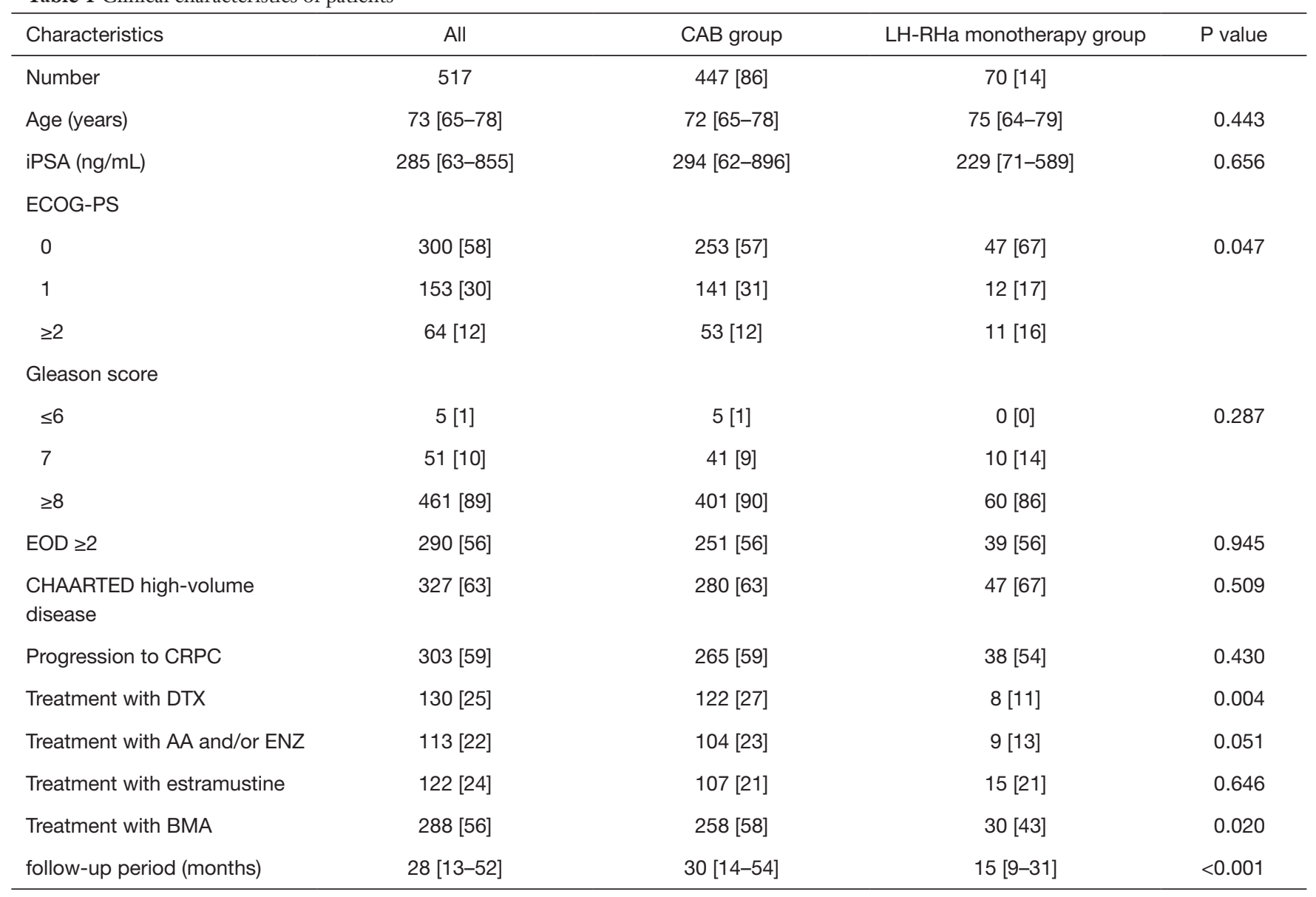

Data are presented as median [IQR] or number [\%]. IQR, interquartile range; iPSA, initial prostate-specific antigen; ECOG-PS, Eastern Cooperative Oncology Group performance status; EOD, extent of disease; CRPC, castration resistance prostate cancer; DTX, docetaxel; $\mathrm{AA}$, abiraterone acetate; ENZ, enzalutamide; BMA, bone-modifying agents.

A

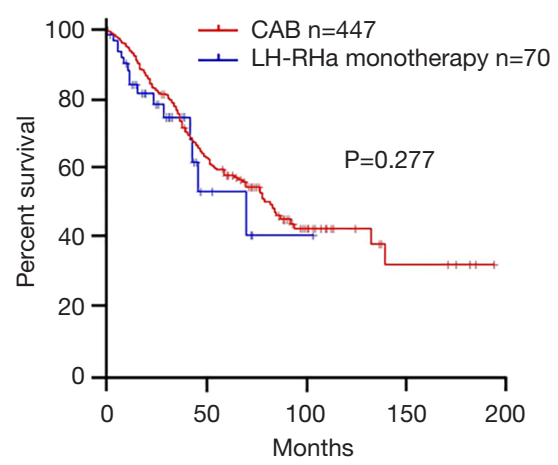

B Cancer-specific survival

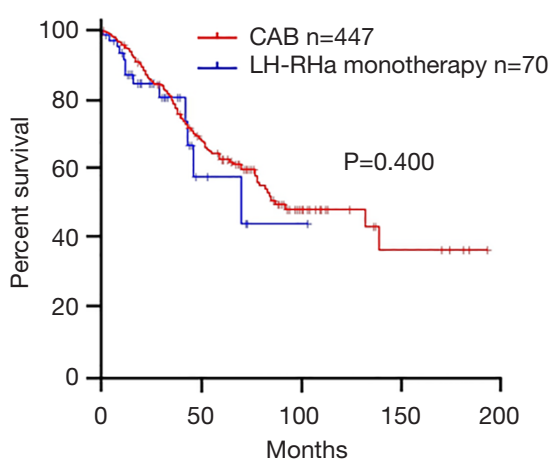

C

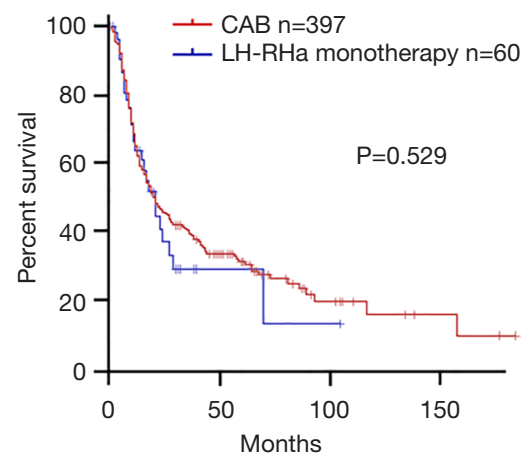

Figure 2 Oncological outcomes between combined androgen blockade therapy (CAB) and luteinizing hormone-releasing hormone analog (LH-RHa) monotherapy. The 5-year overall survival (A), cancer specific survival (B), and castrate-resistant prostate cancer free survival (C) were compared between the CAB and LHRHa monotherapy. 
Table 2 Univariate and multivariate Cox hazard proportional analyses adjusted by inverse probability of treatment weighting (IPTW) model for prognosis

\begin{tabular}{|c|c|c|c|c|}
\hline & Factor & $P$ value & HR & $95 \% \mathrm{Cl}$ \\
\hline Overall survival & $\mathrm{CAB}$ & 0.234 & 0.73 & $0.43-1.23$ \\
\hline Cancer-specific survival & $\mathrm{CAB}$ & 0.346 & 0.76 & $0.43-1.35$ \\
\hline CRPC-free survival & $\mathrm{CAB}$ & 0.247 & 0.81 & $0.57-1.16$ \\
\hline Overall survival & $\mathrm{CAB}$ & 0.442 & 0.79 & $0.45-1.38$ \\
\hline Cancer-specific survival & $\mathrm{CAB}$ & 0.480 & 0.79 & $0.42-1.46$ \\
\hline CRPC-free survival & $\mathrm{CAB}$ & 0.178 & 0.73 & $0.48-1.11$ \\
\hline
\end{tabular}

Inverse probability of treatment weighting analysis. IPTW model included variables; age, iPSA, ECOG-PS, GS, EOD, CHAARTED classification, treatment with DTX, AA and/or ENZ, estramustine, BMA. CRPC, castration resistance prostate cancer; Cl, confidence interval; iPSA, initial prostate-specific antigen; ECOG-PS, Eastern Cooperative Oncology Group performance status; GS, Gleason score; EOD, extent of disease; DTX, docetaxel; AA, abiraterone acetate; ENZ, enzalutamide; BMA, bone-modifying agents.

IPTW-adjusted cox regression model

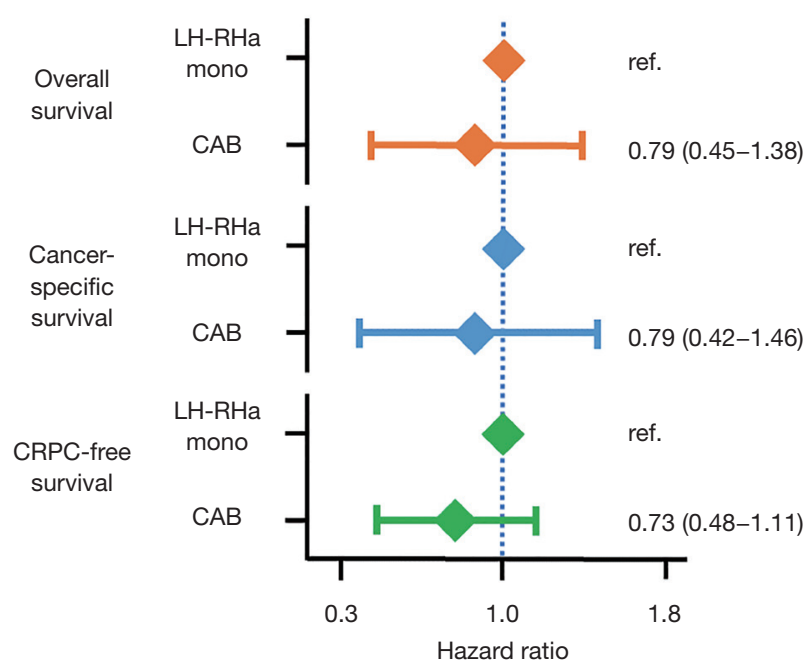

Figure 3 Multivariate Cox hazard proportional analysis adjusted by inverse probability of treatment weighting (IPTW) model for prognosis. IPTW-adjusted multivariate Cox hazard proportional analysis was performed to investigate the effect of combined androgen blockade on overall survival, cancer specific survival, and castrate-resistant prostate cancer free survival. Adjusted variables were age, iPSA, ECOG-PS, GS, EOD, CHAARTED classification, treatment with DTX, AA and/or ENZ, estramustine, BMA. iPSA, initial prostate-specific antigen; ECOG-PS, Eastern Cooperative Oncology Group performance status; GS, Gleason score; EOD, extent of disease; DTX, docetaxel; AA, abiraterone acetate; ENZ, enzalutamide; BMA, bone-modifying agents. 'flare-up' phenomenon" on weak evidence (6). Furthermore, clinicians should also be aware that several studies have failed to prove the existence of "flare-up" phenomenon (27-29). From this point of view, a short-term administration of antiandrogen would be enough to prevent testosterone "flare-up".

Second, the efficacy of CAB in patients with mHNPC can be misleading. Meta-analysis in Western countries (22), RCT in Japan (23), and large-scale observational studies in Japan (24) showed the superiority of CAB in advanced prostate cancer patients (including locally advanced prostate cancer). However, superiority of $\mathrm{CAB}$ was not observed in the subgroup analysis in $\mathrm{mHNPC}$. These results suggested the benefit of $\mathrm{CAB}$ might be limited in the locally advanced prostate cancer alone. Further study is necessary to clarify the superiority of $\mathrm{CAB}$ in those patients.

Recently, upfront therapy with abiraterone or DTX has been recommended for mHNPC with high tumor burden $(16,30)$. However, it remains unclear whether the benefit of $\mathrm{CAB}$ in the mHNPC patients with the high tumor burden. Additional results suggested that effect of $\mathrm{CAB}$ on tumor burden might be limited. There was no significant difference in OS (Figure S2A) and CRPC-FS (Figure S2B) between the CAB and LHRHa monotherapy in the CHAARTED high group. Similarly, no significant difference was observed in OS (Figure S2C) and CRPC-FS (Figure S2D) between the CAB and LHRHa monotherapy in the CHAARTED low group. The IPTW-adjusted multivariate Cox regression analysis showed that OS and 
CRPC-FS were not significantly different between the CAB and LHRHa monotherapy in regardless of tumor burden (Figure S3). However, small sample size in the LHRHa monotherapy group prevent the definitive conclusion. Therefore, further study is necessary for the benefit of $\mathrm{CAB}$ in the era of upfront therapy.

Our study has limitations such as small sample size, short follow-up period, over-weighted effects of minority groups that are far from the center of the patient background of the IPTW method (Figure S4), selection biases for CAB and LH-RHa monotherapy, lack of data of testosterone levels after hormonal therapy, lack of the detailed information on post-treatment, no central review of pathological evaluation, the lack of information on treatment regimen and period of an anti-androgen agent administration, and the lack of ADT-related adverse events evaluation. However, our results suggest the limited efficacy of $\mathrm{CAB}$ over LH-RHa monotherapy in Japanese patients with mHNPC.

In conclusion, we observed no significant differences in oncological outcomes between CAB and LH-RHa monotherapy as initial treatments for patients with mHNPC.

\section{Acknowledgments}

We would like to thank Itsuto Hamano, Teppei Okamoto, Naoki Fujita, Hiromichi Iwamura, Yuki Fujita, Yukie Nishizawa, and the entire staff of the Department of Urology in Hirosaki University for their invaluable help with the data collection. The authors would also like to thank Editage (www.editage.jp) for the English language review.

Funding: This study was supported by Japan Society for the Promotion of Science (JSPS) KAENHI (Grant Numbers of 15H02563, 17K11119, 18K09157, and 19H05556).

\section{Footnote}

Reporting Checklist: The authors have completed the STROBE reporting checklist. Available at http://dx.doi. org/10.21037/tau-20-966

Data Sharing Statement: Available at http://dx.doi. org/10.21037/tau-20-966

Peer Review File: Available at http://dx.doi.org/10.21037/ tau-20-966
Conflicts of Interest: All authors have completed the ICMJE uniform disclosure form (available at http://dx.doi. org/10.21037/tau-20-966). Drs. TN, Shingo H, SN, TS, SK, KM, YA, AI, NT, TH, and CO report grants from Japan Society for the Promotion of Science, during the conduct of the study. The other authors have no conflicts of interest to declare.

Ethical Statement: The authors are accountable for all aspects of the work in ensuring that questions related to the accuracy or integrity of any part of the work are appropriately investigated and resolved. All procedures performed in this study were in accordance with the Declaration of Helsinki (as revised in 2013). The study was approved by institutional ethics board of Hirosaki University school of Medicine (authorization number: 2017-089) and all hospitals approved the protocol. Informed consent was taken from all the patients.

Open Access Statement: This is an Open Access article distributed in accordance with the Creative Commons Attribution-NonCommercial-NoDerivs 4.0 International License (CC BY-NC-ND 4.0), which permits the noncommercial replication and distribution of the article with the strict proviso that no changes or edits are made and the original work is properly cited (including links to both the formal publication through the relevant DOI and the license). See: https://creativecommons.org/licenses/by-nc-nd/4.0/.

\section{References}

1. Kakehi $Y$, Sugimoto M, Taoka R, et al. Evidenced-based clinical practice guideline for prostate cancer (summary: Japanese Urological Association, 2016 edition). Int J Urol 2017;24:648-66.

2. Komura K, Sweeney CJ, Inamoto T, et al. Current treatment strategies for advanced prostate cancer. Int J Urol 2018;25:220-31.

3. Kimura T, Egawa S. Epidemiology of prostate cancer in Asian countries. Int J Urol 2018;25:524-31.

4. Pagliarulo V, Bracarda S, Eisenberger MA, et al. Contemporary role of androgen deprivation therapy for prostate cancer. Eur Urol 2012;61:11-25.

5. National Comprehensive Cancer Network. NCCN clinical practice guidelines in oncology: prostate cancer, 2019, version 1.2019. Available online: https://www.ncen. org/professionals/physician_gls/pdf/prostate.pdf. Accessed 
March 2019.

6. Cornford P, Bellmunt J, Bolla M, et al. EAU-ESTROSIOG Guidelines on Prostate Cancer. Part II: Treatment of Relapsing, Metastatic, and Castration-Resistant Prostate Cancer. Eur Urol 2017;71:630-42.

7. National Institute for Health and Clinical Excellence. Prostate cancer: diagnosis and treatment. NICE; 2008.

8. Hinotsu S, Akaza H, Usami M, et al. Current status of endocrine therapy for prostate cancer in Japan analysis of primary androgen deprivation therapy on the basis of data collected by J-CaP. Jpn J Clin Oncol 2007;37:775-81.

9. Association JU. Clinical Guideline for Prostate Cancer 2016. Tokyo: Kanehara Syuppan; 2016.

10. Narita T, Koie T, Ookubo T, et al. The impact of extended lymph node dissection versus neoadjuvant therapy with limited lymph node dissection on biochemical recurrence in high-risk prostate cancer patients treated with radical prostatectomy: a multi-institutional analysis. Med Oncol 2017;34:1.

11. Okubo T, Mitsuzuka K, Koie T, et al. Two years of bicalutamide monotherapy in patients with biochemical relapse after radical prostatectomy. Jpn J Clin Oncol 2018;48:570-5.

12. Hamano I, Hatakeyama $S$, Narita $S$, et al. Impact of nadir PSA level and time to nadir during initial androgen deprivation therapy on prognosis in patients with metastatic castration-resistant prostate cancer. World J Urol 2019;37:2365-73.

13. Okamoto T, Hatakeyama S, Narita S, et al. Impact of nutritional status on the prognosis of patients with metastatic hormone-naive prostate cancer: a multicenter retrospective cohort study in Japan. World J Urol 2019;37:1827-35.

14. Mitsuzuka K, Arai Y. Metabolic changes in patients with prostate cancer during androgen deprivation therapy. Int J Urol 2018;25:45-53.

15. Okamoto T, Hatakeyama S, Narita S, et al. Validation and development of the CHAARTED criteria in patients with hormone-naïve metastatic prostate cancer: A multiinstitutional retrospective study in Japan. Int J Urol 2020;27:90-1.

16. Sweeney CJ, Chen YH, Carducci M, et al. Chemohormonal Therapy in Metastatic HormoneSensitive Prostate Cancer. N Engl J Med 2015;373:737-46.

17. Scher HI, Morris MJ, Stadler WM, et al. Trial Design and Objectives for Castration-Resistant Prostate Cancer: Updated Recommendations From the Prostate
Cancer Clinical Trials Working Group 3. J Clin Oncol 2016;34:1402-18.

18. Austin PC, Stuart EA. Moving towards best practice when using inverse probability of treatment weighting (IPTW) using the propensity score to estimate causal treatment effects in observational studies. Stat Med 2015;34:3661-79.

19. Cooperberg MR, Hinotsu S, Namiki M, et al. Trans-

Pacific variation in outcomes for men treated with primary androgen-deprivation therapy (ADT) for prostate cancer. BJU Int 2016;117:102-9.

20. Fukagai T, Namiki TS, Carlile RG, et al. Comparison of the clinical outcome after hormonal therapy for prostate cancer between Japanese and Caucasian men. BJU Int 2006;97:1190-3.

21. Samson DJ, Seidenfeld J, Schmitt B, et al. Systematic review and meta-analysis of monotherapy compared with combined androgen blockade for patients with advanced prostate carcinoma. Cancer 2002;95:361-76.

22. Maximum androgen blockade in advanced prostate cancer: an overview of the randomised trials. Prostate Cancer Trialists' Collaborative Group. Lancet 2000;355:1491-8.

23. Akaza H, Hinotsu S, Usami M, et al. Combined androgen blockade with bicalutamide for advanced prostate cancer: long-term follow-up of a phase 3, double-blind, randomized study for survival. Cancer 2009;115:3437-45.

24. Onozawa M, Akaza H, Hinotsu S, et al. Combined androgen blockade achieved better oncological outcome in androgen deprivation therapy for prostate cancer: Analysis of community-based multi-institutional database across Japan using propensity score matching. Cancer Med 2018;7:4893-902.

25. Kahan A, Delrieu F, Amor B, et al. Disease flare induced by D-Trp6-LHRH analogue in patients with metastatic prostatic cancer. Lancet 1984;1:971-2.

26. Kuhn JM, Billebaud T, Navratil H, et al. Prevention of the transient adverse effects of a gonadotropinreleasing hormone analogue (buserelin) in metastatic prostatic carcinoma by administration of an antiandrogen (nilutamide). N Engl J Med 1989;321:413-8.

27. Kotake T, Usami M, Akaza H, et al. Goserelin acetate with or without antiandrogen or estrogen in the treatment of patients with advanced prostate cancer: a multicenter, randomized, controlled trial in Japan. Zoladex Study Group. Jpn J Clin Oncol 1999;29:562-70.

28. Vis AN, van der Sluis TM, Al-Itejawi HHM, et al. Risk of disease flare with LHRH agonist therapy in men with prostate cancer: myth or fact? Urol Oncol 2015;33:7-15. 
29. Krakowsky Y, Morgentaler A. Risk of Testosterone Flare in the Era of the Saturation Model: One More Historical Myth. Eur Urol Focus 2019;5:81-9.

Cite this article as: Narita T, Hatakeyama S, Narita S, Takahashi M, Sakurai T, Kawamura S, Hoshi S, Shimoda J, Kawaguchi T, Ishidoya S, Mitsuzuka K, Arai Y, Ito A, Tsuchiya N, Habuchi T, Ohyama C. Therapeutic effects of the combined androgen blockade therapy versus luteinizing hormonereleasing hormone analog monotherapy in patients with hormone naïve metastatic prostate cancer: a multi-institutional comparative analysis. Transl Androl Urol 2021;10(1):417-425. doi: $10.21037 /$ tau-20-966
30. Fizazi K, Tran N, Fein L, et al. Abiraterone plus Prednisone in Metastatic, Castration-Sensitive Prostate Cancer. N Engl J Med 2017;377:352-60. 


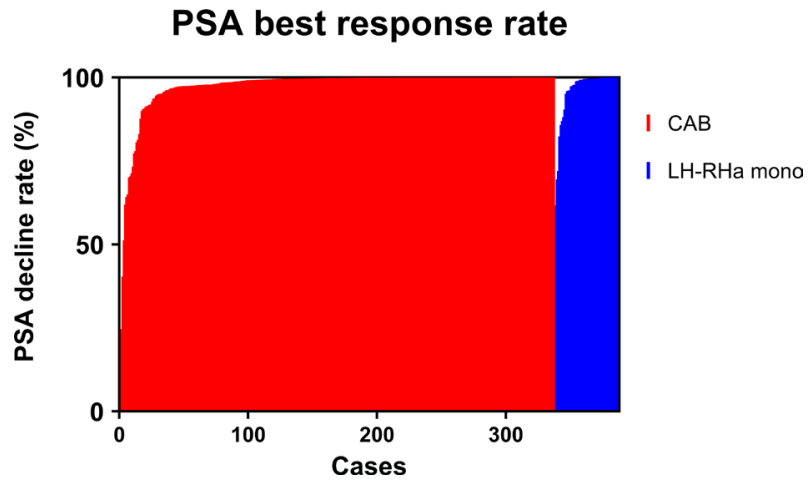

Figure S1 PSA best response rate between combined androgen blockade therapy (CAB) and luteinizing hormone-releasing hormone analog (LH-RHa) monotherapy. Waterfall plots show the best PSA reduction rate with CAB and LH-RHa monotherapy. PSA, prostate-specific antigen.

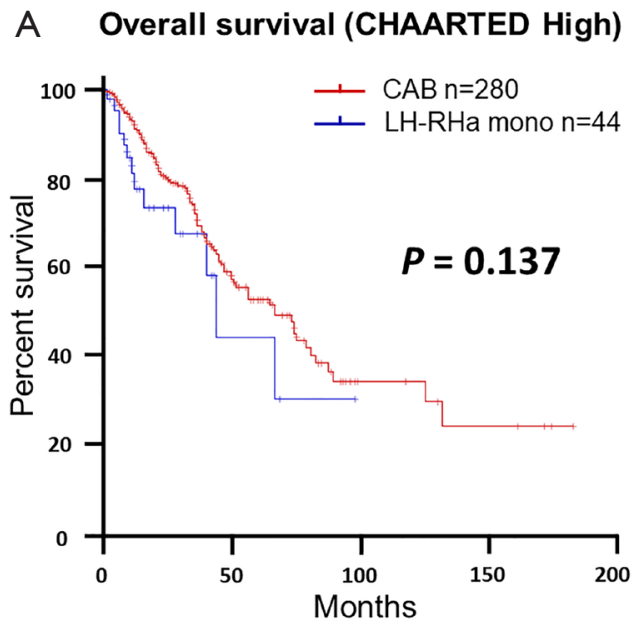

C Overall survival (CHAARTED Low)

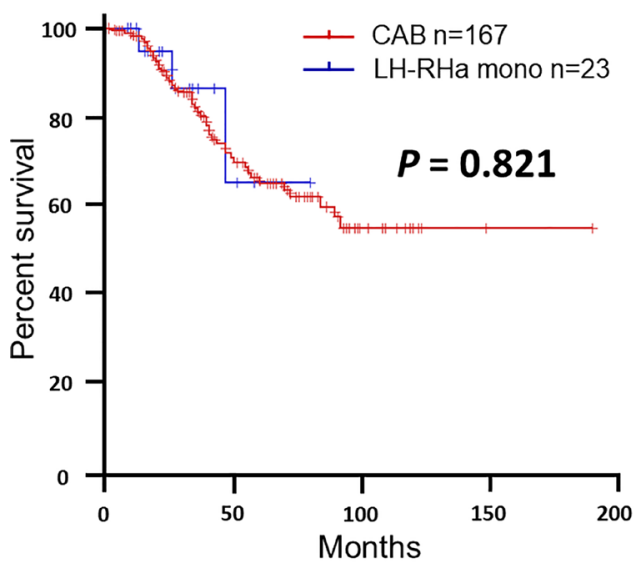

B CRPC-FS (CHAARTED High)

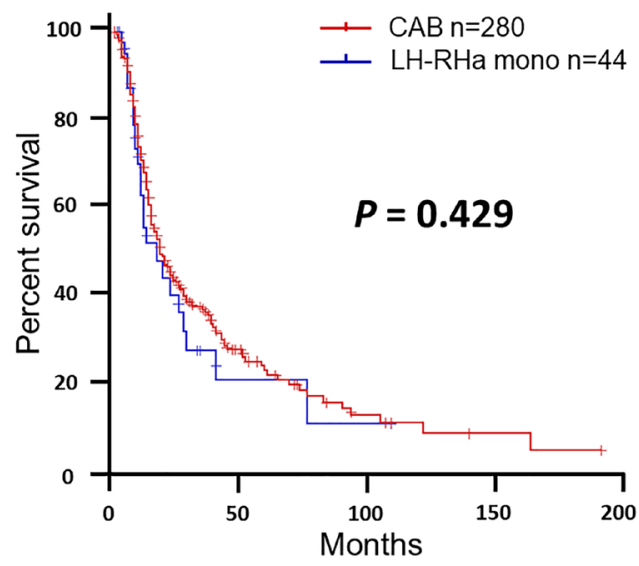

D CRPC-FS (CHAARTED LOW)

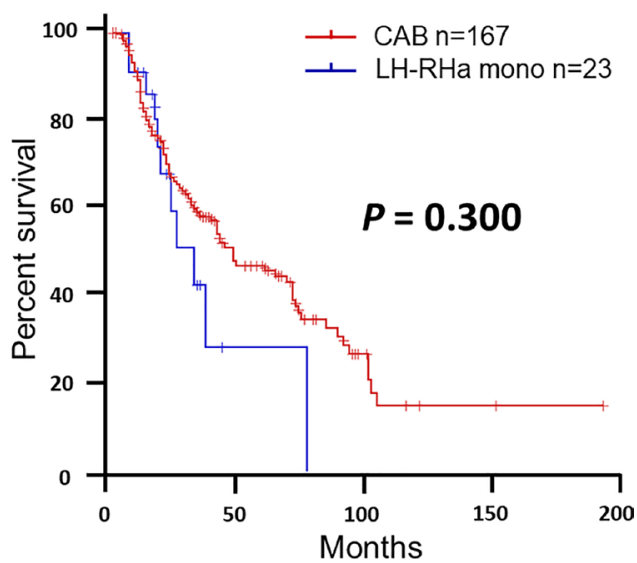

Figure S2 Oncological outcomes between combined androgen blockade therapy (CAB) and luteinizing hormone-releasing hormone analog (LH-RHa) monotherapy by CHAARTED classification. The 5-year overall survival was compared between the CAB and LHRHa monotherapy in the CHAARTED high (A) and low (C). The 5-year CRPC-FS was compared between the CAB and LHRHa monotherapy in the CHAARTED high (B) and low (D). 


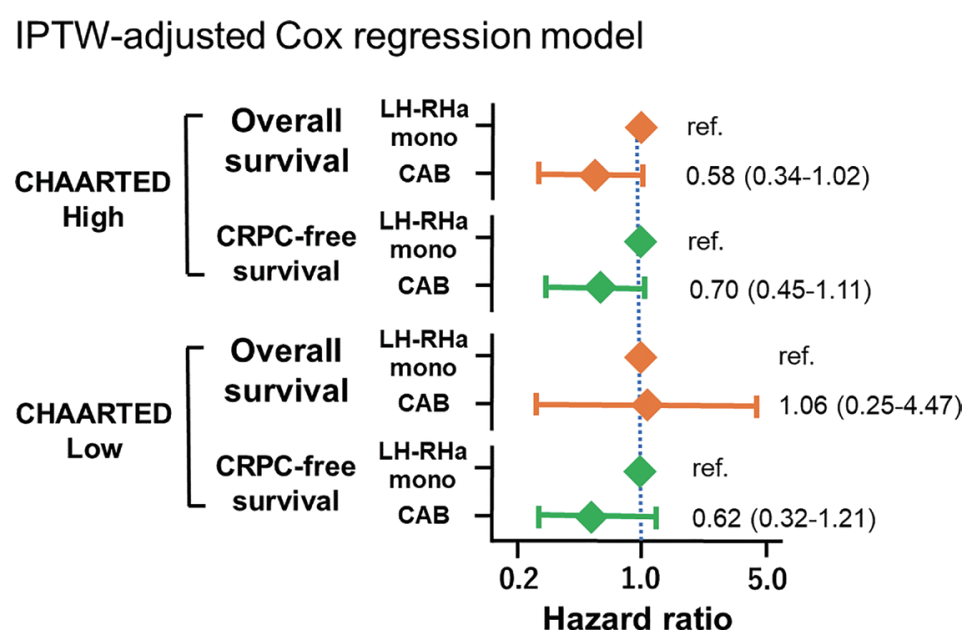

Figure S3 Multivariate Cox hazard proportional analysis adjusted by inverse probability of treatment weighting (IPTW) model for prognosis by CHAARTED classification. IPTW-adjusted multivariate Cox hazard proportional analysis was performed to investigate the effect of combined androgen blockade on overall survival, and castrate-resistant prostate cancer free survival by CHAARTED classification. Adjusted variables were age, iPSA, ECOG-PS, GS, EOD, CHAARTED classification, treatment with DTX, AA and/or ENZ, estramustine, BMA. iPSA, initial prostate-specific antigen; ECOG-PS, Eastern Cooperative Oncology Group performance status; GS, Gleason score; EOD, extent of disease; DTX, docetaxel; AA, abiraterone acetate; ENZ, enzalutamide; BMA, bone-modifying agents.

\section{Weighting by the inverse probability of treatment}

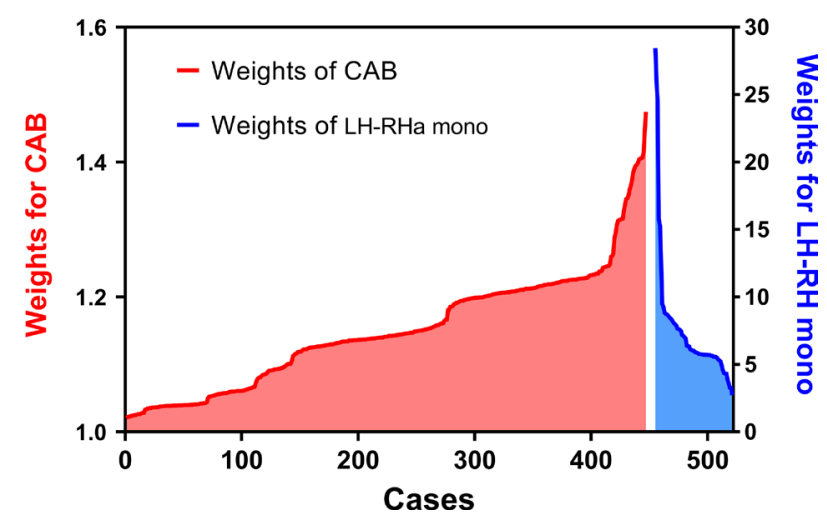

Figure S4 Weighting by the inverse probability of treatment of combined androgen blockade therapy (CAB) and luteinizing hormonereleasing hormone analog (LH-RHa) monotherapy. The weight for treatment between the CAB and LH-RHa monotherapy groups were shown. 